\title{
ADVANCEMENTS IN DRY POWDER INHALER
}

\author{
ADVAIT SHETTY, GANGA SRINIVASAN* \\ Department of Pharmaceutics, Vivekanand Education Society's College of Pharmacy, Hashu Advani Memorial Complex, Chembur (E), \\ Mumbai, Maharashtra, India. Email: ganga.srinivasan@ves.ac.in
}

Received: 24 July 2016, Revised and Accepted: 16 December 2016

\section{ABSTRACT}

The dry powder inhaler (DPI) has become widely known as a very attractive platform for drug delivery. DPIs are being used for the treatment of asthma and chronic obstructive pulmonary disease by many patients. There are over 20 devices presently in the DPI market. DPIs are preferred over nebulizers and pressurized metered dose inhalers. However, some of the challenges of DPI are dependence on inspiratory flow (unsuitable for young children, elderly people), systemic absorption due to deposition of drug in deep lung (unsuitable for local diseases treatment), and increase in upper airway deposition of a large fraction of coarse particles. Hence, there is a need to address these unmet issues. The interpatient variation can be minimized by developing devices independent of patient's inspiratory flow rate or active based powder mechanism. This article reviews DPI devices currently available, advantages of newly developed devices, and formulation technologies. The platform technologies are developed to improve aerosolization and dispersion from the device and decrease the patient related factors. The DPI delivery system has been expanded to treatment of non-respiratory diseases such as migraine and diabetes. The development of innovative DPI device and formulation technologies for delivering therapeutic proteins such as insulin has been accelerated to overcome the problems associated with conventional insulin therapy.

Keywords: Dry powder inhaler, Inspiratory flow rate, Insulin, Platform technologies.

(c) 2017 The Authors. Published by Innovare Academic Sciences Pvt Ltd. This is an open access article under the CC BY license (http://creativecommons. org/licenses/by/4. 0/) DOI: http://dx.doi.org/10.22159/ajpcr.2017.v10i2.14282

\section{PULMONARY DRUG DELIVERY SYSTEM}

Pulmonary route is gaining attention for local or systemic delivery of therapeutic agent because it is non-invasive and bypasses first pass metabolism. Lungs provide a large absorptive surface area, have good blood supply and a thin mucous membrane and hence, can be targeted for delivery of drugs [1]. In 2013, the global market for pulmonary drug delivery technologies reached $\$ 32.4$ billion, with an expectation to grow to $\$ 43.9$ billion till 2018 at a compound annual growth rate (CAGR) of $6.2 \%$. The increased global market share of pulmonary drug delivery is due to the rise in cases of respiratory diseases. Newer technical advancements in the design of pulmonary devices have been applied for the treatment of respiratory disorders such as asthma and chronic obstructive pulmonary disorder as well as non-respiratory conditions like diabetes and migraine [2,3].

Nebulizers, dry powder inhalers (DPI), and metered dose inhalers (MDI) are the three major segments of global pulmonary drug delivery systems market [3]. The DPI is the fastest growing segment in this market, growing at significant CAGR of $12.5 \%$ and is estimated to reach $\$ 31.5$ billion till 2018. Its global market is estimated to overtake the previously dominant segment of MDI by 2018 [2,3].

\section{DPI}

DPI are breath actuated devices which contain powder formulation of micronized drug (approximately below $5 \mu \mathrm{m}$ ) and delivers this medication to the respiratory airways on oral inhalation. The energy emitted from the patient's inspiratory airflow is the driving force for powder dispersion from the device [4].

\section{Why DPIs are preferred over MDIs}

For the last 30 years, the drug delivery to lungs has been dominated by pressurized metered-dose inhalers (pMDIs) as they are believed to constitute more than $80 \%$ of the global market. The most prescribed MDIs have disadvantages both in terms of use and effectiveness [5].
MDIs contain propellants like chlorofluorocarbons that are harmful to the environment [4-6]. MDIs require good coordination between activation and inhalation. Poor coordination is main concern related to proper use of MDIs and causes inefficient therapeutic delivery. The use of pressurized MDIs results in high oropharyngeal deposition and a low amount of drug is delivered to the lungs $[5,6]$. These device require shaking before use to mix the drug and propellant, failure to do so will lead to inefficient drug delivery [5]. The absence of dose counters in these devices also causes a problem as it gets difficult to track the dose taken or remaining $[5,6]$.

DPIs are a better alternative to MDIs as they have many advantages over them. These device are propellant-free and contain micronized drug alone or in combination with a suitable excipient preferably lactose monohydrate. The need for the patient coordination with respect to actuation and inhalation is eliminated as DPIs are breathactivated. This helps in effectively delivering therapeutic agents to the lungs and lower oropharyngeal deposition as compared to MDIs [5,6]. Thorrson et al. (1994) showed that the lung deposition of budesonide in healthy volunteers through the Turbuhaler (DPI) was over twice that through a pMDI (32\% versus $15 \%$, respectively). This suggests that a lower dose of budesonide using the Turbuhaler reduces the systemic side effects and helps achieve the same degree of asthma control [7]. Most of the DPI devices have dose counters that help in detecting the number of doses remaining. As the DPI formulation contains only solid dosage from there is less potential for extractable from components of device [4]. The DPI devices are user-friendly and more convenient to use, hence are preferred by patients over MDIs [5].

\section{Need for advancement in DPI}

To achieve adequate inspiratory flows for reproducible and effective dose delivery, the patients' efforts are important that is determined by DPI's internal resistance. Patients with significant airflow obstruction find high-resistance not suitable as they require greater effort to generate the inspiratory airflow [8]. The area of concern with respect to DPIs is that the dependence on inspiratory flow rate since patients with respiratory 
disorders usually have low inhalation flow rates [4]. Dewar et al. (1999) reported that only $17 \%$ of chronic obstructive airways disease patients achieved flows of $40 \mathrm{l} /$ minute whereas Turbuhaler operates optimally at 50-60 l/minute, hindering its use in elderly and severely ill patients [9].

In the single unit DPI devices, capsules need to be loaded into the device and till the capsule is not empty, the inhalation process is to be carried out, causing high dose variability. Capsules can soften due to high temperatures making them difficult to perforate [5].

Certain multiple unit devices like Diskus are costly to produce due to its complexity and it is also not found to be user-friendly [5].

Multidose DPI devices like Turbuhaler have some issues regarding the feedback to patients, as the patient cannot feel or taste the inhaled particles. There is high dose to dose and device to device variation, and the device cannot be refilled $[5,10]$. The amount of drug released from the DPI devices is reduced under conditions of high humidity due to particle agglomeration [5].

To overcome the problems related to DPIs, there is a need for advancement for better therapeutic control and patient convenience.

\section{Driving forces for the development of new DPIs}

DPI provides a great platform for delivery of new chemical entities or drugs unrelated to respiratory disorder. Many important drugs such as insulin are administered via injection. Drugs or therapeutic agents that are intended for systemic activity for conditions such as cancer, diabetes but are absorbed readily via oral route, can be delivered effectively to the peripheral airways by a DPI [4]

Low-resistance devices do not require a forceful and prolonged inhalation to achieve effective drug delivery as they allow air to flow easily through them easily as compared to high-resistance devices [8]. This suggests that certain high resistance DPI devices make it difficult for children and elderly patients to achieve effective therapeutic action $[4,5]$. Power-assisted dispersion or active powder dispersion can help optimize performance and efficiency [4].

\section{ADVANCEMENTS IN DPI}

Advances in DPI are been made taking into consideration the device as well as the formulation.

Change in device design like making the device flow rate independent. Platform technologies have helped DPI formulation suitable and more efficient in aiding the powder deaggregation from device and increasing the dose deposition in the lungs.

\section{Advancements in device}

New technologies based on passive and active powder dispersion mechanisms are being developed due to the reliance on high inspiratory flow rates for the operation of DPI. The objective is to facilitate deagglomeration of drug particles, resulting in greater lung deposition. The process of particle de-agglomeration can be carried out by developing technologies that use energy as key factor in DPI. Storage of energy in systems based on springs, compressed air or battery powered chambers were some of the alternatives found in inhalers [11]

\section{Spiros inhaler}

Spiros $^{\circledR}$ device from Dura Pharmaceuticals is a DPI that incorporates a battery-powered motor which aerosolizes the drug. The motor gets activated at very low flow rate on inhalation $(5 \mathrm{l} /$ minute). This inhaler is hence helpful in the treatment of critically ill patients, elderly or children. The patient using Spiros inhaler uses less than one-quarter of dispersion energy as compared to Turbuhaler and also there is increased fine particle fraction $[5,11]$.

As shown in Fig. 1 removable circular multiple-dose cassette with 30 wells, in which each well contains a unit dose of micronized albuterol
Table 1: Inhaled insulin DPI systems [30,31]

\begin{tabular}{lll}
\hline Inhalation system & Companies involved & Current status \\
\hline Exubera & $\begin{array}{l}\text { Nektar therapeutics and } \\
\text { pfizer }\end{array}$ & Out of market \\
AIR & Eli lilly and alkermes & No development \\
Afrezza & Mannkind and sanofi & FDA approved \\
\hline
\end{tabular}

DPI: Dry powder inhaler

sulfate and lactose is loaded into the aerosolization chamber by opening the lid and then closing it. The dispersion of aerosol is carried out by aerosolization chamber containing an inspiration-actuated, batterypowered, and twin-blade impeller. During inhalation, ambient air is entrained through holes in the mouthpiece. The inhaler can be reused by replacing the cassettes and it also contains an internal counting mechanism. Since the inhaler uses an active dispersion mechanism, it functions more independently of the patient's inspiratory flow, in contrast with previous DPIs, which relied on inspiratory flow for disaggregation and adequate particle size [12].

\section{Novolizer}

In passive DPIs, the air classifier technology is the most efficient powder dispersion mechanism. Novolizer is a multidose DPI from MEDA. In this inhaler, a cyclone is generated in the mouthpiece through multiple supply channels during inhalation [5,11]. Newman et al. (2000) reported that Novolizer $^{\circledR}$ showed a higher deposition of budesonide in the lung and lower oropharyngeal deposition when compared to Turbuhaler ${ }^{\circledR}[13]$.

It is a multi-use inhalation device containing 200 dose cartridges that can be refilled. This inhaler has unique design is easy to use and with effective performance. Compared to other inhalers, Novolizers have several innovative features offering unique feedback and inhalation control. The patient gets to know whether the powder has been released as he can taste, hear the click in the inhaler and see the "Red" light and the dose indicator on the inhaler confirming correct inhalation which can be seen in Fig. 2. The powder is released only when a certain flow rate $(35 \mathrm{l} /$ minutes) has been achieved, ensuring sufficient drug delivery at the site. This device works at low resistance making it convenient for patients to use the device properly and hence improving compliance $[5,14]$.

A precise and consistent dose over the cartridge lifetime is delivered with minimal dosage variability between actuations. Unlike the Turbuhaler, this inhaler is independent of temperature and humidity $[5,15]$.

\section{Microdose inhaler}

MicroDose Therapeutx has developed MicroDose DPI, an electronic DPI. The powder is deaggregated and aerosolized through a foil dosing blister by a piezo vibrator. The MicroDose DPI is an alternative to GSK's Diskus, as it is reusable for up to 6 months or longer $[4,16]$.

The indicator lights help patients in operation of the device and also give feedback. The "Inhale Now" light tells the user that the device is ready to use and that they are inhaling properly and the "dosing done" light indicates that the dose has been correctly delivered and that the user can stop inhaling, hence give feedback that dose is delivered [16].

The small needles pierce the blisters before dosing to creating openings into the flow channel of the device as can be seen in Fig. 3. When an inhalation sensor detects a threshold level of the patient's inspiratory airflow, activating piezo in turn activating the device. The fine powder that is emitted from the device on inhalation reaches the lungs. As the energy needed to deaggregate and aerosolise the powder is done by piezo vibrator, there is no need for the patient to inhale forcefully. This inhaler operates at low resistance ( $5 \mathrm{l} /$ minute). With this technology, the patient error is eliminated as the inhaler delivers the drug independent of patient coordination, inhalation rate, and orientation $[16,17]$. 


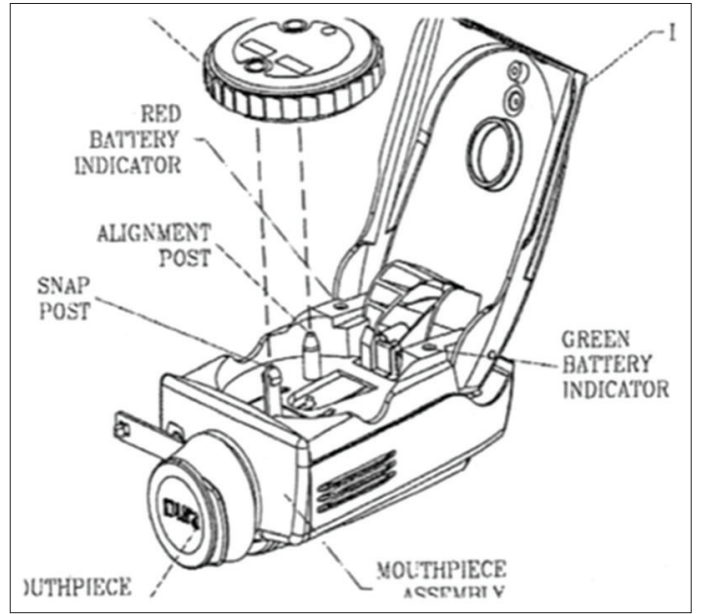

Fig. 1: Spiros inhaler

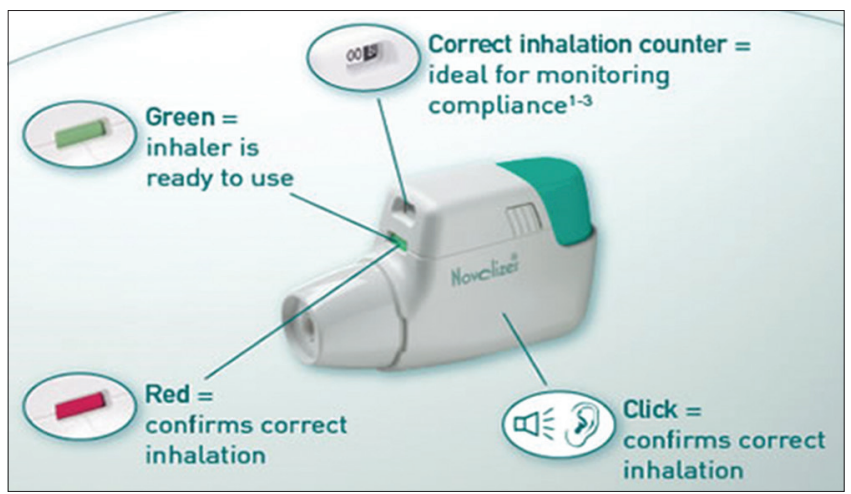

Fig. 2: Novolizer

\section{Direct haler}

In December 2009, Trimel BioPharma SRL acquired the Direct Haler asset of Keldmann Healthcare A/S, which has subsequently been rebranded as TriVair $^{\mathrm{TM}}$. It is an innovative dry power device for intrapulmonary delivery. This device is a single use system that is disposable, pre-metered and pre-filled with pulmonary dose. This device is easy to use and offers effective, accurate, repeatable, and hygienic dosing. The device has been evaluated successfully in the USA, Europe, and India. The performance of DirectHaler Pulmonary is comparable to that of currently marketed DPI device. In addition, the cost-effective device It is the first advanced pulmonary delivery system enabling pharmaceutical companies to carry out manufacturing, filling and packing the device in-house efficiently and it has been found to be cost-effective.

The device is made up of $0.6 \mathrm{~g}$ of polypropylene which consists of a $U$-shaped tube with a double cap that seals each end of the tube and a bend with corrugations. The dose is sealed inside the cap at one end with a laminate foil strip, which can be easily torn off for doseloading into the Powder Whirl chamber, before removing the cap and delivering the dose. This system is useful for powders that are sensitive to temperature or humidity, high drug dose can be used, and deep lung delivery is achieved.

The airflow and powder dispersion are determined by three principles. First, the turbulent air is generated at the air inlet and fed to the Powder Whirl chamber. Second, the PowderWhirl chamber corrugations are designed in such a way that they generate turbulent whirls. These recirculation zones contribute to powder dispersion. Finally, the powder is forced up by the turbulent airflow on the walls of the corrugations and it is then entrained gradually into the inhaled air stream until the device is emptied [18].

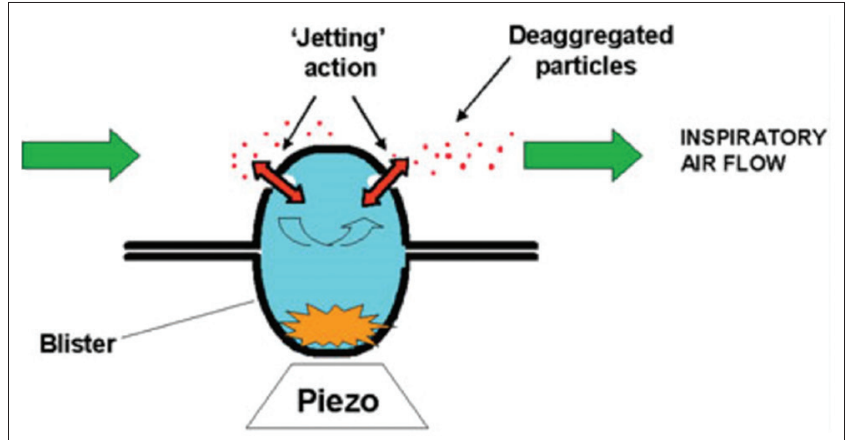

Fig. 3: Schematic representation of powder delivery from MicroDose inhaler [16]

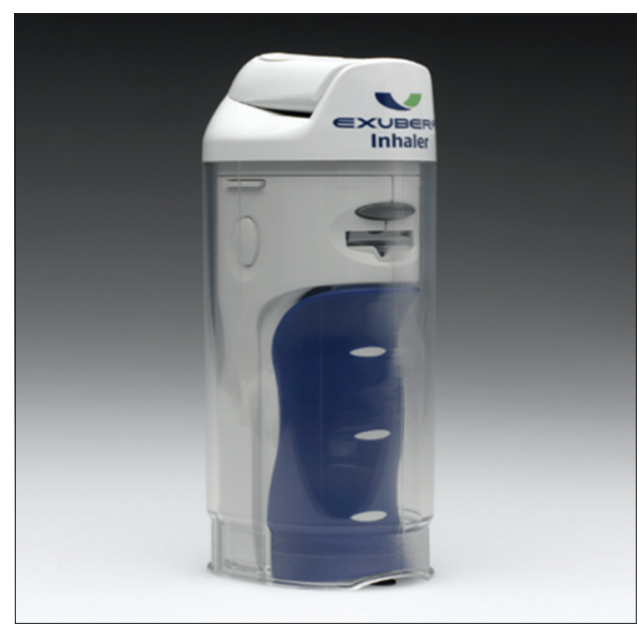

Fig. 4: Exubera ${ }^{\circledR}$ inhaler [31]

Platform technologies

Advancements in these inhalers also include new types of powdered formulations of drugs through the production of porous particles, with low geometric diameter and high potential for lung deposition. Similar porous particles may be coupled to long-sized carrier molecules to reach the lungs with similar efficacy $[11,19]$. Formulation having aerodynamic diameter in range of 1-3 $\mu \mathrm{m}$ results in lower tendency to aggregate, which when combined with lower density particles leads to aerosolization efficiently. Very large particles $(2-3 \mu \mathrm{m})$ deposited in pulmonary region may escape phagocytosis by macrophages and provide sustained delivery and increased systemic bioavailability and can be used to target non-respiratory diseases [20].

Particle engineering modification like particle solubility, surface morphology, and particle chemistry are done to optimize DPI performance. The formulation should deliver accurate, consistent, efficient doses of the drug for effective therapeutic control. Hickey et al. (1994) reviewed that several cohesive and adhesive forces are exerted on powder particles within the device and during aerosol generation. The forces are influenced by Powder characteristics such as packing density, equilibrium moisture content, and particle characteristics such as shape, size, rugosity, crystalline form influences these forces $[4,21]$.

\section{iSPERSE}

iSPERSE $^{\mathrm{TM}}$ has been developed by Pulmatrix. These particles are engineered to provide rapid onset of action, bypass first-pass metabolism and convenience as compared to injection for local drug delivery in lungs and systemic drug delivery. The iSPERSE powders are small in geometric size and relatively dense, yet are highly dispersible and relatively flow rate independent with high emitted doses achieved even at low flow rates [22]. 


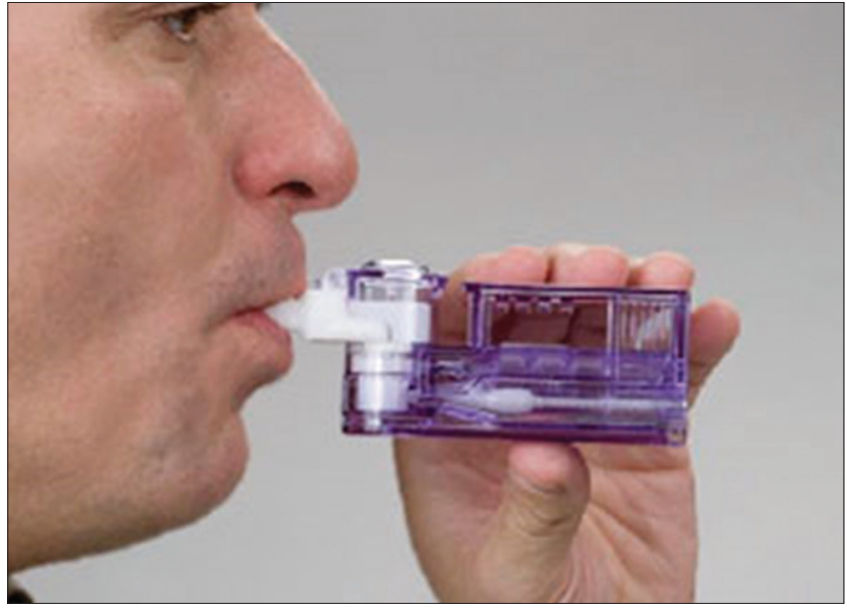

Fig. 5: Afrezza inhaler [34]

High-density, small geometric size and flow rate independent dispersion are some of the characteristics of iSPERSE powders. These powders can be loaded with high or low doses of single or multiple drugs. High emitted dose can be achieved with the help of these particles even at low flow rate. iSPERSE powders are prepared by one-step spray drying. They contain as little as $20 \%$ or less of salts and other excipients in comparison to conventional DPIs containing more than 80-90\% lactose. These powders can help deliver low potent and large molecules by oral inhalation $[22,23]$.

\section{Powder hale ${ }^{\circledR}$}

This technology developed by Vectura Group utilize a special component called "Force Control Agents" (FCA). Examples of patent protected FCAs include magnesium stearate and L-leucine. This component coats lactose and/or drug substance to reduce the interactive forces acting upon them. This proprietary technique offers optimal flow properties as well as superior de-aggregation and aerosolization characteristics. Low dose variation with high lung penetration of inhaled particles, dose uniformity, and enhanced product stability are achieved by PowderHale technology. This technology has helped deliver a consistent fine particle dose as compared to conventional DPI formulations having a restricted penetration to the lungs. A wide variety of therapeutic drugs and inhaler devices use the PowderHale technology. Ultribro and Seebri Breezhaler utilize this technology [24].

\section{PulmoSphere}

These are spray dried, small, porous particles containing phospholipids. The drug is dissolved in a solvent and then precipitated to produce fine particles $[25,26]$. This technique allows for greater control of particle properties like morphology, porosity, density, particle size and distribution, which further govern powder properties such as powder flow and dispersibility [27]. Dispersible powders with improved aerodynamic properties have been achieved without the addition of carrier particles; the powder properties are improved. High drug loadings about $90-95 \% \mathrm{w} / \mathrm{w}$ are possible [25]. Improvements in lung delivery can be achieved by spray dried, low density and highly porous particles which decrease interparticle cohesive forces [27]. The PulmoSpheres delivery is independent of the patient's inspiratory flow rate, reducing dosing variability. TOBI Podhaler which utilizes PulmoSphere technology is indicated for the management of cystic fibrosis patients with Pseudomonas aeruginosa [25].

\section{TechnoSphere}

MannKind's Technosphere technology allows for pulmonary route administration of drugs that are administered only via injection. Processing steps like milling, sizing, blending or micronizing are not needed once, Technosphere particles are formed by either controlled crystallization to form crystalline particles or spray drying to give amorphous particles. These particles are made from novel excipient fumaryl diketopiperazine (FDKP), and the technology is based on the $\mathrm{pH}$-induced intermolecular self-assembly of FDKP. These particles are homodisperse, with a mean geometric diameter of about $2 \mu \mathrm{m}$ and after inhalation dissolve rapidly. High surface area and high internal porosity are some of the features of these particles [28,29].

Technosphere formulations are prepared from drugs with molecular weights ranging from $500 \mathrm{Da}$ (small molecule) to $140,000 \mathrm{Da}$ (monoclonal antibodies) and FDKP with consistent and narrow particle size range. Drugs of anionic, cationic, hydrophilic, and hydrophobic nature, as well as proteins and peptides, have been formulated successfully with the help of this technology. This formulation achieves excellent bioavailability as there is rapid systemic absorption that mimics intra-arterial circulation. It also provides advantages like bypassing the first pass metabolism and degradation in peripheral circulation. Many of these formulations have been administered via pulmonary route in rat models for systemic delivery [28]. Afrezza uses this technology to deliver insulin by oral inhalation $[28,29]$.

\section{INHALED INSULIN}

Diabetes is characterized by high blood glucose level which is caused when pancreas fail to produce enough insulin (Type 1 diabetes) or when cells of the body do not respond properly to the insulin produced (Type 2 diabetes). Type 1 patients require insulin administration immediately whereas Type 2 patients require it at a later stage. The insulin delivery systems that are being used currently by patients are considered as invasive and noncompliant. In order overcome these issues, companies are developing approaches to delivery insulin by routes which are noninvasive, reduce the pain and patient compliance [30]. Delivery of insulin through pulmonary route, i.e., by oral inhalation has become an alternative to the conventional subcutaneous injection for the management of diabetes as lungs provide a large surface area and large blood supply. Inhaled insulin was first investigated by Gänsslen in 1925 by using a nebulizer system, the effect on blood glucose was noted bioavailability was low and limitations of the device made the route impractical $[30,31]$.

The ideal insulin inhaler should be portable, user-friendly and achieve optimal glycemic control. Inhaled insulin delivery by powder devices may provide a considerable benefit in quality of life for a large number of patients [11]. Several companies as listed in Table 1 have worked to develop inhaled insulin systems for diabetic patients over the last two decades. Inhaled insulin bioavailability for each of the devices varies in the range of $10 \%$ to $46 \%$ since much of the drug is lost within the device or due to deposition in the upper airways [31].

Nektar Therapeutics and Pfizer collaborated to develop an insulin inhaler, Exubera ${ }^{\circledR}$ (see Fig. 4). It was approved by the Food and Drug Association (FDA) and the European Medicines Agency for the treatment of both types of diabetes in 2006. The dry powder formulation containing $1 \mathrm{mg}$ or $3 \mathrm{mg}$ of regular human insulin was packaged in blister packets. These unit doses are equivalent to 3 units and 8 units of subcutaneously delivered short-acting insulin, respectively, and are delivered by a mechanical inhaler which depends on patient's inspiratory flow rate. Pfizer withdrew Exubera ${ }^{\circledR}$ from the market due to poor sales and acceptance in October 2007 [30,31].

Eli Lilly and Co. and Alkermes Inc. developed AIR ${ }^{\circledR}$ insulin system. The insulin in dry powder form is delivered via a breath actuated mechanical inhaler. The dry powder insulin formulation contained doses of $6 \mathrm{mg}$ and $9 \mathrm{mg}$ which were equivalent to 2 and 6 units of subcutaneously delivered insulin. They were packed in capsules. The inhaled particles are less dense as compared to other systems but have large particle size of about 5 to $30 \mu \mathrm{m}$ and delivered to the alveolar space efficiently. Eli Lily are not pursuing development of this product even though this system has been through extensive phase III clinical trial [31].

Afrezza developed by Mannkind Corp. was approved by the U.S. FDA approved in 2014 (see Fig. 5) [32]. It uses the Technosphere platform 
technology [29,31]. Afrezza consists of a dry powder formulation of human insulin delivered from MedTone ${ }^{\circledR}$ inhaler to help patients achieve blood sugar control as the powder is aerosolized and delivered to the lung. It is a rapid-acting inhaled insulin, having short duration of action to improve glycemic control in adults with Types 1 and 2 diabetes. It is administered at the beginning of each meal [32,33]. The Technosphere insulin delivers the insulin directly into the arterial circulation with excellent systemic action as they are rapidly absorbed after inhalation [29]. Afrezza is available in two different strengths identified by color like 4 units (blue cartridge) and 8 units (green cartridge) and both dispensed in foil packages [33].

\section{CONCLUSION}

Over the last few years, due to growth in cases of respiratory diseases, innovations in formulations, combinations and unique design of inhalers have led to growth of this delivery system. The DPI market is full of innovative and unique device designs for inhalers, but no inhaler fulfill all the requirements of an ideal DPI. A novel DPI should not only efficiently deliver drugs to the patients but also be reliable and convenient to use. The advanced research in field of DPIs will make formulations incorporating matrix and drugs like proteins and peptides to achieve to achieve intracellular drug delivery and specific pulmonary drug deposition. The nature, safety of carrier, drug type, device, site of action, and disease state will determine the carrier choice. Health professions and physicians have a great range of options available for treatment of respiratory and nonrespiratory disorders as sophisticated formulations efficient pulmonary delivery devices have become available.

\section{REFERENCES}

1. Chaturvedi NP, Solanki H. Pulmonary drug delivery system: Review. Int J Appl Pharm 2013;5(3):7-10.

2. Pulmonary Drug Delivery Systems: Technologies and Global Market. Available from: http://www.prnewswire.com/news-releases/ pulmonary-drug-delivery-systems technologies-and-globalmarkets-262529191.html.

3. Pulmonary Drug Delivery Systems Market to Benefit from Growing Prevalence of COPD and Asthma. Transparency Market Research. Available from: http://www.transparencymarketresearch.com/ pressrelease/pulmonary-drug-delivery-systems.htm. [Last posted on 2015 Mar 13].

4. Ashurst II, Malton A, Prime D, Sumby B. Latest advances in the development of dry powder inhalers. Res Focus 2000;3(7):246-56.

5. O'Connor BJ. The ideal inhaler: Design and characteristics to improve outcomes. Respir Med 2004;98 Suppl A: S10-6.

6. Geller DE. Comparing clinical features of the nebulizer, metered-dose inhaler, and dry powder inhaler. Respir Care 2005;50(10):1313-21.

7. Thorsson L, Edsbäcker S, Conradson TB. Lung deposition of budesonide from Turbuhaler is twice that from a pressurized metereddose inhaler P-MDI. Eur Respir J 1994;7(10):1839-44.

8. Colthorpe P, Voshaar T, Kieckbusch T, Cuoghi E, Jauernig J. Delivery characteristics of a low-resistance dry-powder inhaler used to deliver the long-acting muscarinic antagonist glycopyrronium. J Drug Assess 2013;2(1):11-6.

9. Dewar MH, Jamieson A, McLean A, Crompton GK. Peak inspiratory flow through Turbuhaler in chronic obstructive airways disease. Respir
Med 1999;93(5):342-4.

10. Burnell PK, Small T, Doig S, Johal B, Jenkins R, Gibson GJ. Ex-vivo product performance of Diskus and Turbuhaler inhalers using inhalation profiles from patients with severe chronic obstructive pulmonary disease. Respir Med 2001;95(5):324-30.

11. Muchão FP, Filho LV. Advances in inhalation therapy in pediatrics. J Pediatr (Rio J) 2010;86(5):367-76.

12. Rau JL. The inhalation of drugs: Advantages and problems. Respir Care 2005;50(3):367-82.

13. Newman SP, Pitcairn GR, Hirst PH, Bacon RE, O'Keefe E, Reiners M, et al. Scintigraphic comparison of budesonide deposition from two dry powder inhalers. Eur Respir J 2000;16(1):178-83.

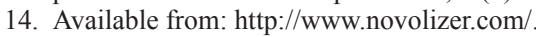

15. Fyrnys B, Stang N, Wolf-Heuss E. Stability and performance characteristics of a budesonide powder for inhalation with a novel dry powder inhaler device. Curr Opin Pulm Med 2001;7 Suppl 1:S7-11.

16. Brown BA, Rasmussen JA, Becker DP, Friend DR. A piezo-electronic inhaler for local and systemic applications. Drug Deliv Technol 2004;4(8):90-3.

17. Corcoran TE, Venkataramanan R, Hoffman RM, George MP, Petrov A, Richards T, et al. Systemic delivery of atropine sulfate by the microdose dry-powder inhaler. J Aerosol Med Pulm Drug Deliv 2013;26(1):46-55

18. Available from: http://www.trivairdevice.com.

19. Son YJ, McConville JT. Advancements in dry powder delivery to the lung. Drug Dev Ind Pharm 2008;34:948-59.

20. Edwards DA, Ben-Jebria A, Langer R. Recent advances in pulmonary drug delivery using large, porous inhaled particles. J Appl Physiol 1998;85(2):379-85.

21. Hickey AJ, Concessio NM, Van Oort MM, Platz RM. Factors influencing the dispersion of dry powders as aerosols. Pharm Technol 1994;18(8):58-64.

22. Available from: http://www.pulmatrix.com/isperse_platform.html.

23. Sung JC, Manzanedo D, Ku AT, Kenyon J, Trautman BG, DeHaan WH, et al. iSPERSE ${ }^{\mathrm{TM}}$ : Formulation and in vitro characterization of a novel dry powder drug delivery technology. Respir Drug Deliv Eur 2011;2:411-14

24. Available from: http://www.vectura.com.

25. Geller DE, Weers J, Heuerding S. Development of an inhaled drypowder formulation of tobramycin using PulmoSphere ${ }^{\mathrm{TM}}$ technology. J Aerosol Med Pulm Drug Deliv 2011;24(4):175-82.

26. Weers J, Tarara T. The PulmoSphere ${ }^{\mathrm{TM}}$ platform for pulmonary drug delivery. Ther Deliv 2014;5:277-95.

27. Weers JG, Bell J, Chan HK, Cipolla D, Dunbar C, Hickey AJ, et al. Pulmonary formulations: What remains to be done? J Aerosol Med Pulm Drug Deliv 2010;23 Suppl 2:S5-23.

28. Available from: http://www.mannkindtechnologies.com

29. Angelo R, Rousseau K, Grant M, Leone-Bay A, Richardson P. Technosphere insulin: Defining the role of Technosphere particles at the cellular level. J Diabetes Sci Technol 2009;3:545-54.

30. Al-Tabakha MM, Arida AI. Recent challenges in insulin delivery systems: A review. Indian J Pharm Sci 2008;70(3):278-86.

31. Mastrandrea LD. Inhaled insulin: Overview of a novel route of insulin administration. Vasc Health Risk Manag 2010;6:47-58.

32. FDA Approves Afrezza to Treat Diabetes. Available from: http://www. fda.gov/NewsEvents/Newsroom/PressAnnouncements/ucm403122. htm.

33. Available from: https://www.afrezza.com

34. Tenderich A. Just how hot are we for inhalable insulin? January, 2011. Available from: http://www.healthline.com/diabetesmine/just-how-hotare-we-for-inhalable-insulin\#1 